\title{
Retention in care under universal antiretroviral therapy for HIV-infected pregnant and breastfeeding women ('Option B+') in Malawi
}

Lyson Tenthani $i^{*, a, b, c}$, Andreas D. Haas ${ }^{*, b}$, Hannock Tweya ${ }^{\text {b,d,e }}$, Andreas Jahn ${ }^{\mathrm{a}, \mathrm{c}}$, Joep J. van Oosterhout ${ }^{\mathrm{f}}$, Frank Chimbwandira ${ }^{\mathrm{a}}$, Zengani Chirwaa, ${ }^{\mathrm{a}, \mathrm{c}}$, Wingston $\mathrm{Ng}^{\prime} \mathrm{ambi}^{\mathrm{d}}$, Alan Bakali, Sam Phiri, Landon Myer ${ }^{\mathrm{h}}$, Fabio Valeri ${ }^{\mathrm{b}}$, Marcel Zwahlen ${ }^{\mathrm{b}}$, Gilles Wandeler ${ }^{\mathrm{b}, \mathrm{i}, \mathrm{i}}$, Olivia Keiser ${ }^{*, b}$, for the Ministry of Health in Malawi and leDEA Southern Africa

\section{See related paper on page 599}

\begin{abstract}
Objective: To explore the levels and determinants of loss to follow-up (LTF) under universal lifelong antiretroviral therapy (ART) for pregnant and breastfeeding women ('Option B+') in Malawi.

Design, setting, and participants: We examined retention in care, from the date of ART initiation up to 6 months, for women in the Option B+ program. We analysed nationwide facility-level data on women who started ART at 540 facilities $(n=21939)$, as well as individual-level data on patients who started ART at 19 large facilities $(n=11534)$.

Results: Of the women who started ART under Option B+ $(n=21939), 17 \%$ appeared to be lost to follow-up 6 months after ART initiation. Most losses occurred in the first 3 months of therapy. Option B + patients who started therapy during pregnancy were five times more likely than women who started ART in WHO stage $3 / 4$ or with a CD $4^{+}$cell count 350 cells/ $\mu$ or less, to never return after their initial clinic visit [odds ratio (OR) 5.0, 95\% confidence interval (Cl) 4.2-6.1]. Option B+ patients who started therapy while breastfeeding were twice as likely to miss their first follow-up visit (OR 2.2, 95\% Cl 1.8-2.8). LTF was highest in pregnant Option B+ patients who began ART at large clinics on the day they were diagnosed with HIV. LTF varied considerably between facilities, ranging from 0 to $58 \%$. Conclusion: Decreasing LTF will improve the effectiveness of the Option B + approach. Tailored interventions, like community or family-based models of care could improve its effectiveness. (c) 2014 Wolters Kluwer Health | Lippincott Williams \& Wilkins
\end{abstract}

AIDS 2014, 28:589-598

Keywords: antiretroviral therapy, loss to follow-up, option $\mathrm{B}+$, pregnancy, prevention of mother-to-child transmission/vertical transmission retention in care

\footnotetext{
${ }^{a}$ Department of HIV and AIDS, Ministry of Health, Lilongwe, Malawi, ${ }^{b}$ Institute of Social and Preventive Medicine, University of Bern, Switzerland, 'International Training and Education Centre for Health/Department for Global Health, University of Washington, Seattle, USA, 'Lighthouse Trust Clinic, Lilongwe, Malawi, ${ }^{\mathrm{e}}$ The International Union Against Tuberculosis and Lung Disease, Paris, France, ${ }^{\mathrm{f}}$ Dignitas International, Zomba, ${ }^{\mathrm{g}}$ Baobab Trust, Lilongwe, Malawi, ${ }^{\mathrm{h}}$ Division of Epidemiology \& Biostatistics, School of Public Health \& Family Medicine, University of Cape Town, South Africa, 'Department of Infectious Diseases, University Hospital Bern, Switzerland, and 'Department of Infectious Diseases, University of Dakar, Dakar, Senegal. Correspondence to Olivia Keiser, PhD, Institute of Social and Preventive Medicine (ISPM), Finkenhubelweg 11, 3012 Bern, Switzerland.
}

Tel: +413163135 15; e-mail: okeiser@ispm.unibe.ch

* Lyson Tenthani, Andreas D. Haas and Olivia Keiser contributed equally to the writing of this article.

Received: 25 June 2013; revised: 8 November 2013; accepted: 8 November 2013. 


\section{Introduction}

Programmes that effectively prevent mother-to-child transmission (PMTCT) of HIV can reduce the rate of transmission under $5 \%$, and reduce morbidity and mortality in both mothers and children [1]. In 2010, the WHO recommended lifelong antiretroviral therapy (ART) for women who were eligible for treatment and who had $\mathrm{CD}^{+}$cell counts 350 cells/ $\mu$ l or less, or in WHO clinical stage 3 or 4 . For women not yet eligible for treatment, WHO recommended two alternative PMTCT strategies for short-term antiretroviral prophylaxis. Option A started women on antepartum zidovudine (AZT) from 14 weeks after pregnancy, as well as on lamivudine (3TC) and nevirapine (NVP) during labour, followed by a zidovudine (ZDV)/3TC tail for 7 days, with daily infant NVP during breastfeeding. Option B started women on triple-drug prophylaxis 14 weeks after gestation, and continued throughout pregnancy and breastfeeding $[2,3]$. These strategies depend on $\mathrm{CD}^{+}{ }^{+}$cell testing to determine women's eligibility for lifelong ART [4]. In a systematic review on sub-Saharan African PMTCT programmes, $\mathrm{CD} 4^{+}$cell count testing was identified as a major barrier to PMTCT [5].

In 2011, the Malawian Ministry of Health (MOH) adopted a pragmatic public health approach to improve the low PMTCT coverage in Malawi and implemented a modified Option B, commonly referred to as Option B+ [6]. Option $\mathrm{B}+$ provides universal lifelong ART for all HIV-infected pregnant and breastfeeding women, regardless of $\mathrm{CD}^{+}$cell count and/or WHO clinical stage. The policy was designed for the Malawian healthcare system with its limited laboratory capacity, and population with high HIV prevalence, short birth intervals, and extended breastfeeding. It avoids $\mathrm{CD} 4^{+}$cell count testing and continues ART even after women discontinue breastfeeding $[7,4]$.

Malawi's initiative has sparked interest and debate among the international public health community [4,8-11], and the latest 2013 WHO guidelines recommend lifelong ART for all pregnant and breastfeeding women [12]. Option B+ may improve PMTCT and may also reduce maternal morbidity and mortality [13], as well as transmission between serodiscordant partners [14]. But concerns about implementation, patient acceptability and retention in care must be addressed [8].

Results of routine monitoring and evaluation (M\&E) of the Malawi HIV programme suggest that Option $\mathrm{B}+$ is feasible and acceptable. The new PMTCT guidelines increased antiretroviral coverage among pregnant women from $49 \%$ in 2011 to $60 \%$ in 2012 [15]. Programmatic data indicate that most women who started ART with an Option $\mathrm{B}+$ indication were still in care after 6 months $(82.6 \%)$, and most of them were still in care after 12 months $(76.9 \%)[6,16]$. The exact timing and predictors of loss to follow-up (LTF) across different settings are unknown.

We describe retention in care for women who started ART under Option B + using routine facility-level M\&E data and patient-level data from a subset of facilities with an electronic medical record system (EMRS).

\section{Methods}

Two complementary data sources were used to explore facility and patient-level factors associated with LTF. We first provide a summary of the Malawi ART/PMTCT programme, and then describe the data sources, eligibility criteria, definitions and statistical analysis separately for facility and patient-level analyses.

\section{Malawi National antiretroviral therapy/ prevention of mother-to-child transmission programme}

The scale-up of free ART started in 2004 using a simplified protocol and standard ART regimens. It relied mainly on clinical monitoring for toxicity and treatment failure. ART patients are followed monthly for the first 6 months and every 2-3 months thereafter. PMTCT was implemented in 2002: single-dose NVP was given to the mother at the onset of labour and to the infant after birth. In 2007, the WHO 'AZT combination prophylaxis' regimen was rolled out, initially at district hospitals and larger facilities. Similar to 'Option A', this regimen provides daily ZDV for the mother during pregnancy with an additional dose of 3TC and NVP during labour, and a 1-4-week prophylaxis with ZDV-syrup for the infant $[2,17]$. When Option B+ was introduced in September 2011, pregnant and breastfeeding women began to receive a simple fixed-dose combination of tenofovir, 3TC and efavirenz [7]. Patients lost to followup are not actively traced.

\section{Facility-level data: eligibility, definitions and statistical analysis}

Each quarter, representatives from the $\mathrm{MOH}$ visit all ART/PMTCT facilities in the country. They review the treatment cards of all patients who started ART between two and three quarters preceding the review, and extract treatment outcomes at 6 and 12 months on therapy. ART outcomes for women who started with an Option B+ indication are aggregated by facility. Routine M\&E methods are described in detail in the $\mathrm{MOH}$ quarterly HIV Programme Reports [6,18]. We included all Option B+ patients who started ART between October 2011 and March 2012 at 540 facilities (Fig. 1). Patients who transferred between facilities were excluded to prevent double-counting.

Our primary outcome was the proportion of patients 'lost to follow-up' 6 months after they began ART. In 
Facility-level data

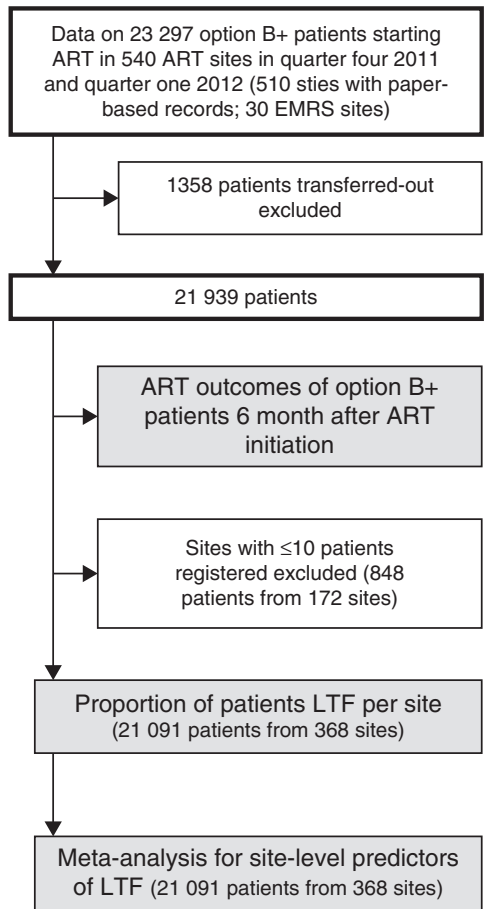

Individual-level data

158855 patients from 20 sites with EMRS

Excluded patients:

One site because of poor data quality $(n=21074)$

Male patients ( $n=53$ 057)

Non-naive patients or patients not on ART $(n=1955)$

Patients aged $<16$ years $(n=6833) ;>50$ years $(n=5158)$

Patients started ART before the implementation of option $\mathrm{B}+(01 / 09 / 2011)(n=59$ 151)

Patients with unknown reason for starting ART $(n=68)$

Patients with unknown ART outcome: $(n=25)$

11534 eligible patients from 19 sites with EMRS (table 1)

Figure 3: Time from HIV testing to ART initiation

Figure 2: ART outcomes since introduction of option $\mathrm{B}+: n=11534$

ART inititation $<3$ month before closure of database $(n=2810)$

Table 2: Predictors of no follow-up $(n=8724)$

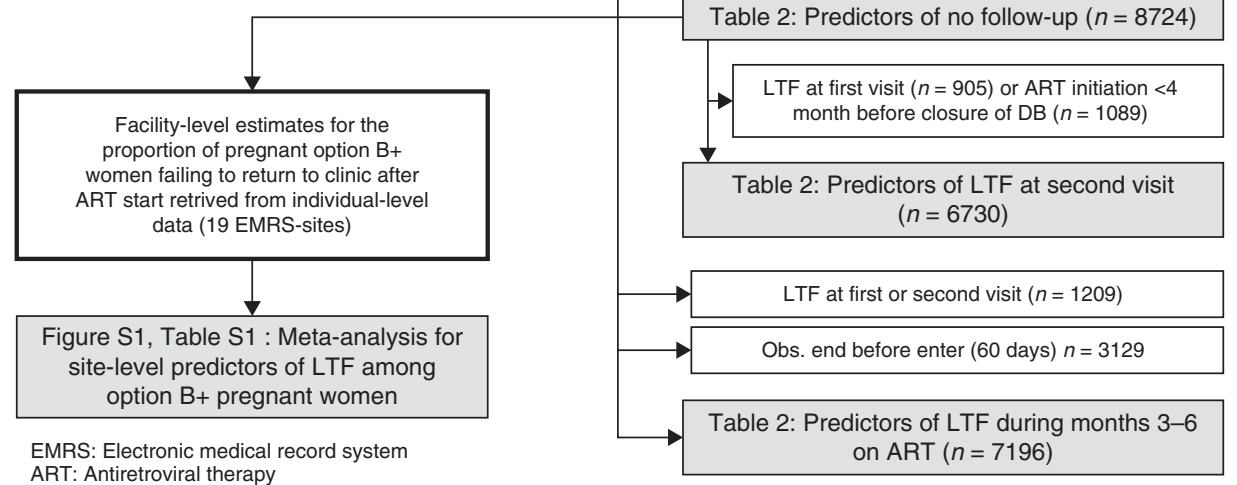

ART: Antiretrovic therapy system LTF: Loss to follow-up

Fig. 1. Inclusion of facilities and patients in different analyses.

facility-level data, we defined patients who missed an appointment and did not return to care for more than 60 days as lost to follow-up. We calculated the proportion of patients lost to follow-up in large facilities, which have EMRS, and in smaller ART facilities, which have no EMRS. For facilities where more than 10 patients were registered (Fig. 1), we calculated the site-specific log odds to get LTF and used random-effects meta-regression to explore between-site variability. Results were transformed to odds.

\section{Patient-level data: eligibility, definitions and statistical analysis}

At larger facilities, individual-level data are collected with an EMRS during patient consultation. The EMRS system was introduced in 2006. It guides healthcare workers through treatment protocols and stores key data for patient care [19]. Quality checks are implemented in the system and manual checks are performed monthly. We extracted patient-level data from all 20 facilities in Central and Southern Malawi in which EMRS was in place when the Option B + programme began. Data from five facilities in Northern Malawi were not extracted because they used a different database system with known data quality problems. Of the 20 eligible facilities, one was excluded because of serious data quality problems. Nineteen facilities provided reliable data and were included.

We included all treatment-naive women aged $16-50$ years who started ART in the 19 facilities (Fig. 1) between September 2011 (the date Option B+ was implemented) and the date of the most recent follow-up visit in a given 
facility (the closing date). We excluded women whose reason for starting ART was unknown.

Our primary outcomes were 'no follow-up visit after ART initiation' and LTF. We defined patients who missed their first follow-up visit and did not return to care for more than 60 days as 'no-follow up after ART initiation' on the day of the missed appointment. Patients who missed a subsequent follow-up visit and did not return to care for more than 60 days were classified as lost to follow-up. We distinguished between no follow-up and LTF by assuming that a woman's failure to return for the first follow-up visit might reflect her resistance to starting ART, whereas we regarded her failure to return thereafter as a problem of retention. Secondary outcomes included the time from HIV diagnosis to ART initiation, death, transfer out and stopped ART. We compared patient outcomes across three groups, based on the indication for ART: 'ART for own health' (non-pregnant female patients in WHO clinical stage 3 or 4 or with $\mathrm{CD}^{+}$cell count $\leq 350 / \mu \mathrm{l}$ ); 'B+ pregnant' (pregnant women starting ART regardless of $\mathrm{CD}^{+}{ }^{+}$cell count or clinical stage); or 'B + breastfeeding' (breastfeeding women who start ART regardless of $\mathrm{CD}^{+}{ }^{+}$cell count or clinical stage).

We plotted cause-specific cumulative incidence functions for all ART outcomes by patient group. To examine factors associated with no follow-up and LTF, we used univariable and multivariable logistic regressions with a random effect for the cohorts. We used competing risk regression to examine variables associated with LTF after the second follow-up visit. This model accounts for the interdependence of LTF and competing events [20,21]. Models were adjusted for age $(<30,30-39, \geq 40$ years) and facility type (health centre, mission, district or central hospital). Patients were followed from initiation of ART to the date of the outcome of interest, or were censored at database closure or because they had completed 6 months of follow-up. We collected additional information on service delivery and ART preparation for the 19 EMRS facilities to explore the association between these factors and no follow-up visit among pregnant Option B+ women. For each facility, we assessed the percentage of $\mathrm{B}+$ pregnant women who never came back after starting ART and estimated the underlying mean percentage by combining estimates for facilities in random-effect metaanalysis. Results are shown in a forest plot. Meta regression was used to study facility-level factors associated with no follow-up visit among pregnant Option B+ patients. To show differences in the delay of ART initiation among patients groups, we plotted Kaplan-Meier curves. All analyses were done in Stata version 11 (Stata Corp., College Station, Texas, USA).

\section{Ethical approval}

Ethical approval was granted by the Malawian Institutional Review Board, the National Health Sciences Research Committee (No: 962).

\section{Role of the funding source}

The funders had no role in study design, data collection and analysis, decision to publish, or preparation of the manuscript.

\section{Results}

\section{Facility-level data}

Included in the analysis were a total of 21939 patients who started ART under Option B+ at one of 540 facilities from October 2011 to March 2012 and who did not transfer to another facility $(n=1358$ patients who transferred out were excluded). At 6 months after ART initiation, $17984(82.0 \%)$ were retained in care; 3749 (17.1\%) were lost to follow-up; 151 (0.7\%) were known to have died and $53(0.2 \%)$ were known to have stopped ART. In EMRS sites, LTF was $22 \%$ and thus higher than in other, usually smaller, facilities with paper-based records in which $15.6 \%$ of all patients were lost to followup. LTF varied considerably between 0 and $58 \%$ at the 368 facilities with more than 10 Option $\mathrm{B}+$ patients registered. Overall, 137 (37.2\%) facilities performed well $(<10 \%$ of all patients lost to follow-up 6 months after ART initiation). LTF was moderate (10-19\%) at 108 (29.4\%) facilities and high $(>20 \%)$ at $123(33.4 \%)$ facilities.

There was no clear association between facility-level predictors and LTF. There was, however, a tendency towards higher LTF in urban facilities than in rural facilities [odds ratio (OR) 1.4, 95\% confidence interval (CI) 1.0-2.0], in $\mathrm{MOH}$-managed facilities than in faith-based, private and other facilities (OR 1.2, 95\% CI 1.0-1.5), in central hospitals than in district hospitals and health centres (OR 2.7, 95\% CI 0.97.9), and in larger facilities with EMRS than in smaller facilities with paper-based records (OR 1.3, 95\% CI $0.9-1.8)$.

\section{Patient-level data}

We analysed ART outcomes for 11534 women who started ART at the 19 large EMRS facilities since September 2011 (see Table 1 for baseline characteristics). Patients were followed for a total of 3501 person-years, for a median [interquartile range (IQR)] duration of $3.3(1.0-5.9)$ months. We compared across patient groups the patients' risk of not returning to the clinic after the first visit or getting lost to follow-up thereafter. Compared to patients who started ART for their own health, Option $\mathrm{B}+$ patients who started ART while pregnant were five times more likely to fail to return to the clinics after the initial visit (adjusted OR 5.0, 95\% CI 4.2-6.1) and patients who started treatment while breastfeeding were twice as likely to miss their first follow-up visit (adjusted OR 2.2, 95\% CI 1.8-2.8) (Table 2, Fig. 2). A similar pattern was seen for LTF at the second visit, 
Table 1. Baseline characteristics of all female patients aged 16-50 years starting antiretroviral therapy (ART) since 1 September 2011 in 19 facilities with electronic medical record systems.

\begin{tabular}{|c|c|c|c|c|}
\hline & Total & $\begin{array}{l}\text { Non-pregnant } \\
\text { women starting } \\
\text { ART for their } \\
\text { own health }\end{array}$ & $\begin{array}{l}\text { Pregnant women } \\
\text { starting ART } \\
\text { due to Option B+ }\end{array}$ & $\begin{array}{c}\text { Breastfeeding women } \\
\text { starting ART due } \\
\text { to Option B+ }\end{array}$ \\
\hline Number of patients & 11534 & 6177 & 3320 & 2037 \\
\hline Median (IQR) age (years) & $30.4(25.9-35.8)$ & $33.1(28.3-38.8)$ & $27.9(23.8-31.7)$ & $28.4(24.6-32.5)$ \\
\hline$<30(\%)$ & $5466(47.4)$ & $2058(33.3)$ & $2143(64.6)$ & $1265(62.1)$ \\
\hline $30-39(\%)$ & $4658(40.4)$ & $2835(45.9)$ & 1109 (33.4) & $714(35.1)$ \\
\hline$\geq 40(\%)$ & $1410(12.2)$ & $1284(20.8)$ & $68(2.1)$ & $58(2.9)$ \\
\hline \multicolumn{5}{|c|}{ ART eligibility due to $\mathrm{CD}^{+}$cell count $(\%)$} \\
\hline Eligible & $2853(24.7)$ & $2731(44.2)$ & $39(1.2)$ & $10(0.5)$ \\
\hline Not eligible & $117(1.0)$ & $50(0.8)$ & $112(3.7)$ & $28(1.4)$ \\
\hline Missing $^{\mathrm{a}}$ & $8564(74.3)$ & $3396(55.0)$ & $3169(95.5)$ & $1999(98.1)$ \\
\hline \multicolumn{5}{|l|}{ WHO clinical stage (\%) } \\
\hline Stage 1 & $3072(26.6)$ & $2052(33.2)$ & $1020(30.7)$ & $0(0.0)$ \\
\hline Stage 2 & $494(4.3)$ & $434(7.0)$ & $60(1.8)$ & $0(0.0)$ \\
\hline Stage 3 & 3336 (28.9) & $3239(52.4)$ & $97(2.9)$ & $0(0.0)$ \\
\hline Stage 4 & $465(4.0)$ & $452(7.3)$ & $13(0.4)$ & $0(0.0)$ \\
\hline Missing & $4167(36.1)$ & $0(0.0)$ & $2130(64.2)$ & $2037(100.0)$ \\
\hline \multicolumn{5}{|c|}{ ART eligible due to $\mathrm{CD}^{+} \leq 350 / \mu \mathrm{l}$ or WHO stage $3 / 4(\%)$} \\
\hline Eligible & $6370(55.2)$ & $6142(99.4)$ & $218(6.6)$ & $10(0.5)$ \\
\hline Not eligible or missing & $5164(44.8)$ & $35(0.6)$ & $3102(93.4)$ & $2027(99.5)$ \\
\hline HIV testing date missing (\%) & $1355(11.8)$ & $827(13.4)$ & $288(8.7)$ & $240(11.8)$ \\
\hline \multicolumn{5}{|l|}{ Year of ART start (\%) } \\
\hline 2011 & $5735(49.7)$ & $2901(47.0)$ & $1590(47.9)$ & $1244(61.1)$ \\
\hline 2012 & $5799(50.3)$ & $3276(53.0)$ & $1730(52.1)$ & $793(38.9)$ \\
\hline Number (\%) pregnant & $3320(28.8)$ & $0(0.0)$ & $3320(100.0)$ & $0(0.0)$ \\
\hline \multicolumn{5}{|l|}{ Most frequent ART regimen (\%) } \\
\hline d4T 3TC NVP & $5819(50.5)$ & $5479(88.7)$ & $179(5.4)$ & $161(7.9)$ \\
\hline TDF 3TC EFV & $5420(47.0)$ & 488 (7.9) & $3090(93.1)$ & 1842 (90.4) \\
\hline
\end{tabular}

3TC, lamivudine; d4T, stavudine; EFV, efavirenz; IQR, interquartile range; NVP, nevirapine; TDF, tenofovir.

${ }^{a}$ ART eligibility assessment due to $\mathrm{CD}^{+}$cell count was missing if no CD4 ${ }^{+}$cell count was measured between 3 months before and 1 month after start of ART.

although the level was much lower. Between months 3 and 6, LTF was low and similar across groups (Table 2). Six months after ART initiation, the proportion of women who had no follow-up visit after ART initiation or were lost to follow-up reached $23.9 \%(95 \% \mathrm{CI}$ 22.6-25.3\%) in all Option B+ patients, 29.4\% (95\% CI $27.6-31.3 \%)$ in $\mathrm{B}+$ pregnant women, $16.1 \%(95 \% \mathrm{CI}$ $14.3-18.0)$ in $\mathrm{B}+$ breastfeeding women and $9.6 \%(95 \%$ CI $8.7-10.6 \%)$ in women with a low $\mathrm{CD}^{+}{ }^{+}$cell count or advanced clinical stage.

The proportion of women known to have died or transferred to other clinics at 6 months after ART initiation was higher among women who started ART for their own health (Fig. 2) than among those who started under Option B+. Documented mortality among Option $\mathrm{B}+$ patients was low. Mortality over a period of 6 months was $0.4 \%(95 \%$ CI $0.2-0.7 \%)$ for $\mathrm{B}+$ pregnant women, $0.5 \%$ (95\% CI $0.2-0.9 \%)$ for $\mathrm{B}+$ breastfeeding women and $3.0 \%$ (95\% CI 2.6-3.6\%) for women who started ART for their own health. After controlling for age and facility type, Option $\mathrm{B}+$ patients who started ART on the day of HIV testing were almost twice as likely never to return to the clinic (adjusted OR 1.9, 95\% CI 1.5-2.4) than were Option B + patients who started ART later $(P<0.001)$.
Among pregnant Option $\mathrm{B}+$ patients (the group with the highest risk of no follow-up after ART initiation) the proportion of women who had no follow-up visit after ART initiation varied from 1.2 to $42.5 \%$ between facilities. Much of this variation could be explained by facility-level factors: facilities with the largest proportions of $\mathrm{B}+$ pregnant women had the largest proportion of these women with no follow-up visits (Figure S1, http:// links.lww.com/QAD/A452). Facilities that offered additional adherence counselling to that required by national guidelines (e.g. peer counselling by mother mentors) had lower rates of early LTF (Table S1, http:// links.lww.com/QAD/A452).

An analysis of time from HIV diagnosis to ART initiation included a total of 10179 patients. Over a third (35\%) of all pregnant Option $\mathrm{B}+$ patients with a known HIV testing date began ART on the day they were diagnosed ( $n=1072 / 3032 ; n=288$ missing HIV testing date). The median (IQR) time from HIV diagnosis to ART initiation was $9(0-64)$ days for pregnant Option $\mathrm{B}+$ patients, 141 (16-351) days for breastfeeding Option B+ patients and $76(15-480)$ days for patients who started for their own health (Fig. 3). Results were similar when we restricted the analysis to facilities with less than 5\% missing values in HIV testing date (data not shown). 


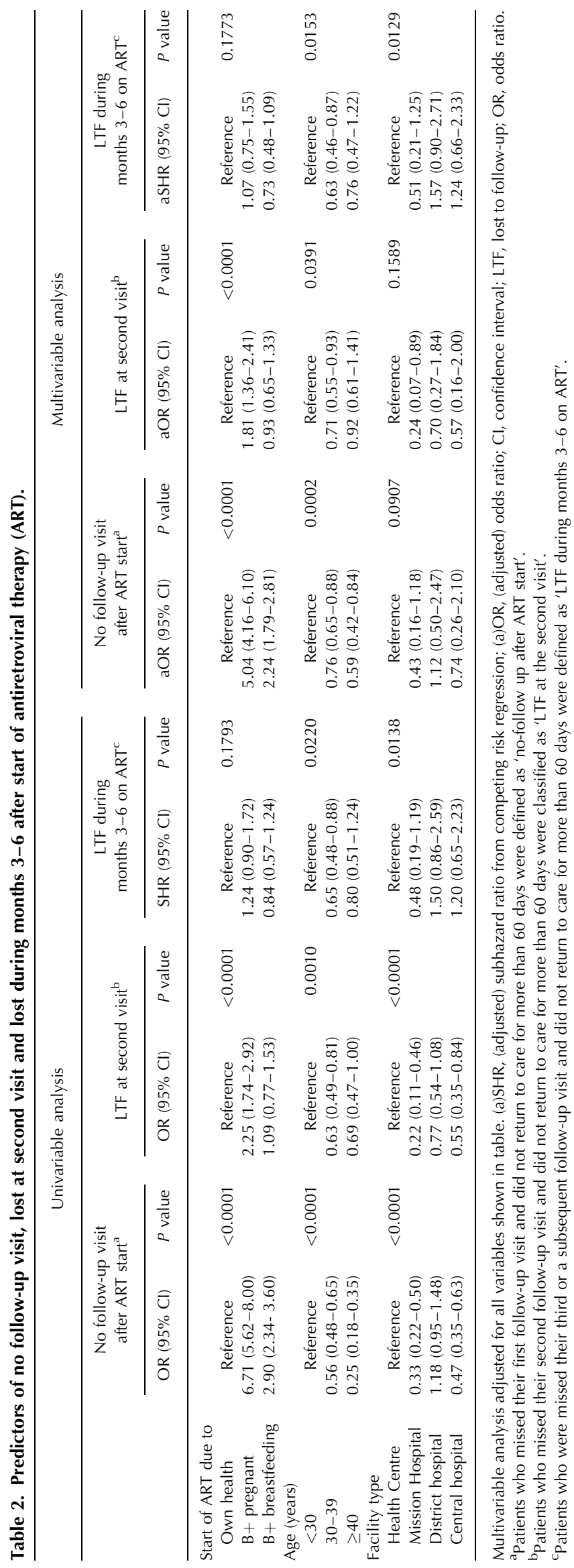


(a)

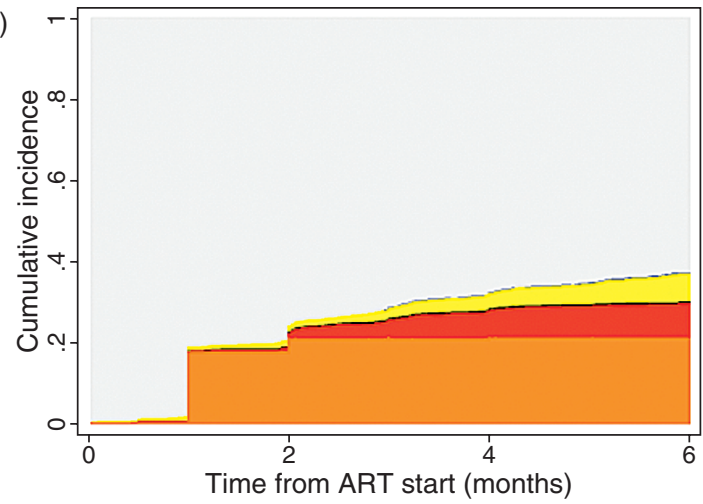

(b)

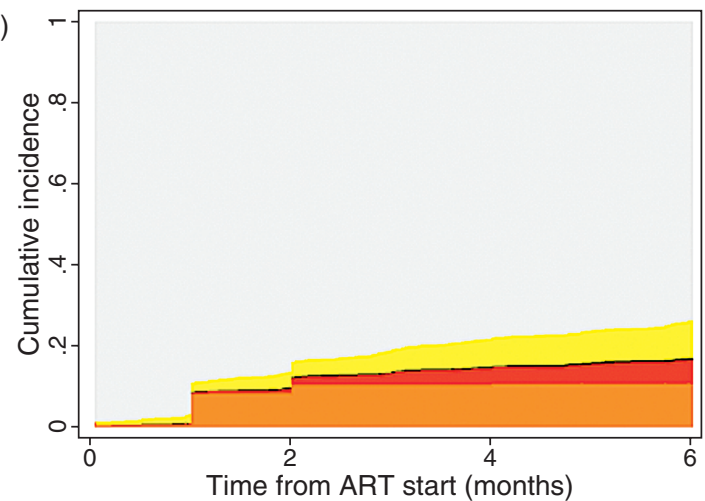

(c)

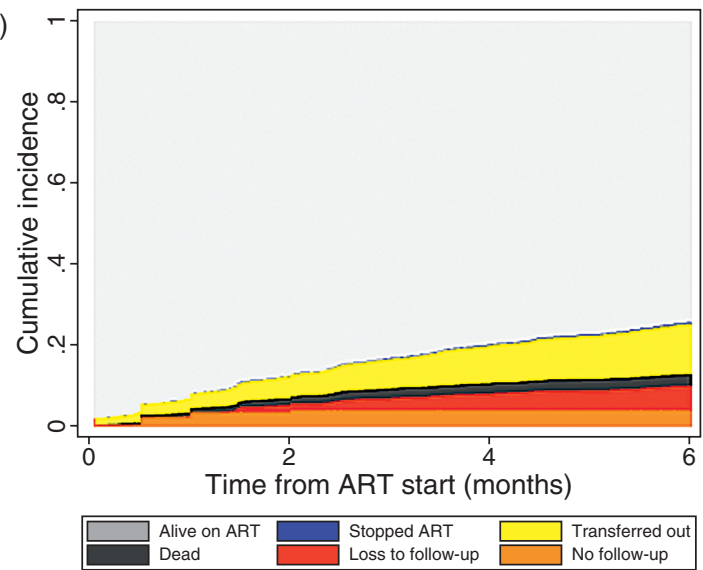

Fig. 2. Cumulative incidence of outcomes on antiretroviral therapy (ART) since the introduction of Option B+. The panels show the cumulative incidence of treatment outcomes over time from the start of ART in months. Treatment outcomes are compared across three groups based on patients' indication for ART: Option B+ indication and ART start during pregnancy $(\mathrm{a})$, Option $\mathrm{B}+$ indication and ART start after delivery while breastfeeding (b) and WHO stage 3 or 4 and/or $\mathrm{CD}^{+}{ }^{+} 350 / \mu$ l or less (c). Estimates are derived from competing risk regression.

\section{Discussion}

Under Option $\mathrm{B}+$, on average, $17 \%$ of all women were lost to follow-up 6 months after ART initiation. In the 19 large facilities with EMRS and available patient-level

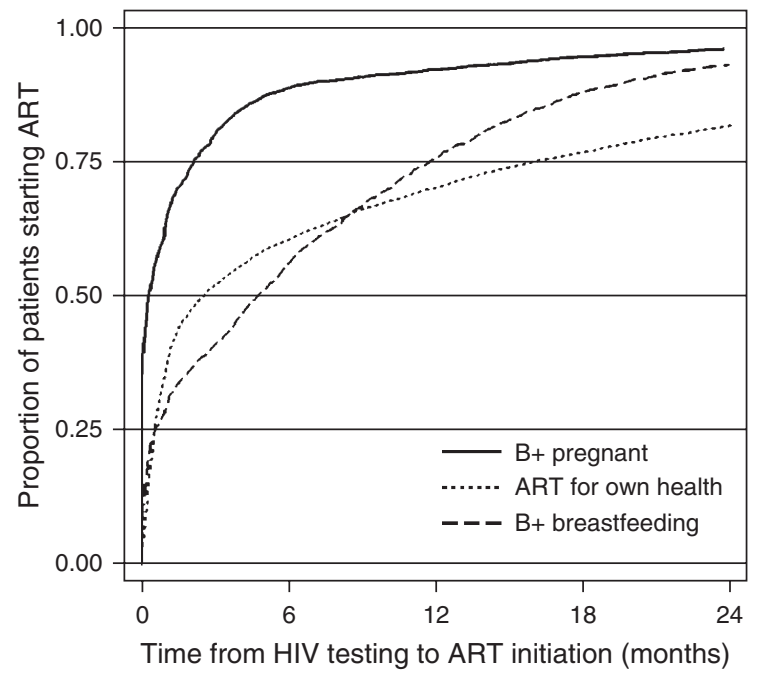

Fig. 3. Time from HIV testing to initiation of antiretroviral therapy (ART). Kaplan-Meier functions for the time from HIV testing to initiation of ART among patients who started ART. Separate functions are shown for patient who started ART with Option B+ indication during pregnancy ('Option

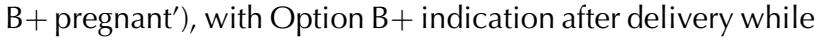
breastfeeding ('Option B+ breastfeeding') and in WHO clinical stage 3 or 4 and/or $\mathrm{CD}^{+} 350 / \mu$ l or less ('ART for own health').

data, we found higher LTF (24\%). Compared to women who initiated ART for their own health, women who started ART to prevent MTCT during pregnancy were five times more likely never to return to the clinic after they initiated ART. Women who began ART while breastfeeding were twice as likely to not return compared to those who started for their own health. More than onethird of all pregnant Option B+ patients initiated ARTon the day of diagnosis, and members of this group were almost twice as likely never to return to the facilities after the initial visit as pregnant Option $\mathrm{B}+$ patients who started later. There was extensive variation in the level of LTF between clinics, ranging from 0 to $58 \%$ at 6 months after ART initiation. Notably, the risk of LTF was highest in facilities where the patient cohort contained a large proportion of Option $\mathrm{B}+$ patients.

Our results confirm and extend previous studies on retention in care under Option B+ in Malawi. In these studies, 17 and $22 \%$ of all women who started ART with an Option $\mathrm{B}+$ indication were lost to follow-up 6 and 12 months after ART initiation [6,16]. The 7\% point higher rate of LTF that we found in larger facilities with available patient-level data may be caused by healthsystem factors affecting retention (e.g. overburdened staff, ability to capture and report data), which may be less favourable in larger ART programmes [22]. A recent study conducted at a large urban maternity unit in Malawi also reported high initial LTF and a retention rate below the national average [23]. But it is also possible that 
misclassification of patients (e.g. unaccounted transfers) as lost to follow-up is more common in larger facilities: these facilities are located in more urban regions with a mobile population.

There are several possible reasons Option $\mathrm{B}+$ patients are more likely to be lost to follow-up than those who start ART for their own health. Some women may find PMTCT intervention coercive [24]. High dropout rates could reflect women's resistance to starting ART. Some early dropouts may never have started ART, or have stopped after the first dose in the health facility. It is also possible that Option B+ women may have been less well prepared for ART, and thus less likely to adhere to treatment and to attend follow-up visits $[25,26]$. Our findings of better retention in care in patients who did not start ART on the day of diagnosis, and in those who received additional counselling may support this idea. However, the reasons and consequences for differences in the timing of ART initiation require further investigation. More time to prepare for ART initiation may also be the reason why patients who started ART while breastfeeding may have had more time to prepare for ART than those who initiated ART before delivery. This may have lowered the former's risk of LTF. Women who start ART immediately do not have the chance to disclose their HIV status to spouses or relatives, and prior disclosure may improve ART adherence [25]. On the contrary, non-disclosure was frequent among pregnant women who feared stigmatization, divorce and physical violence even before Option $\mathrm{B}+$ was implemented $[27,28]$; and there is limited empirical evidence to document the advantages of systematically delayed ART [29]. Finally, patients who are lost to follow-up may have died or transferred to another facility without EMRS documentation and thus have been misclassified as lost to follow-up [30]. Unaccounted transfers are a common problem in Malawi [31], but under-recording of death is unlikely to be common in $\mathrm{B}+$ pregnant women, given the relative good health of HIV-infected pregnant women. High LTF and poor PMTCT coverage have been described under Option A and B [5]; many of the factors causing poor retention may therefore not be specific to Option B+, but a general problem of PMTCT.

The strength of this analysis is that it combines insights from a large longitudinal patient-level dataset with those from facility-level data with national coverage. We were able to evaluate differences in LTF across specific categories of women, including Option $\mathrm{B}+$ women who started ART before and after delivery. We analysed patient-level data and described predictors of LTF during different points after ART initiation. Precise HIV testing date allowed us to describe time elapsed between HIV testing, ART initiation and clinical outcomes.

The study has also several limitations. We lacked information on date of delivery, parity, gestational age at ART initiation, duration of breastfeeding and relevant health-system indicators. Although we evaluated associations between some facility and patient-level characteristics and patient outcomes, we could not rely on information about psychosocial and structural determinants of retention in care (e.g. coercion and resistance to starting ART). The short length of follow-up duration and the fact that our data were confined to adult patients on ART prevented us from assessing potential long-term benefits of Option B+ like decreased child morbidity and mortality. In Malawi, electronic data entry and monitoring does not start when positive HIV+ status is determined and women become eligible for ART. Instead, data collection begins at time of ART initiation, a condition that limited our ability to assess PMTCT coverage under Option B+. Finally, patients who transferred to another facility had to be excluded from facility-level data to avoid double counting. The analysis of facility-level predictors of LTF may have been biased by the exclusion of these patients because the proportion of transfers varied greatly between facilities.

In summary, in the first months after Option $\mathrm{B}+$ was implemented in Malawi, the number of pregnant and breastfeeding women who started lifelong ART substantially increased, and the time between the first positive HIV test and treatment start decreased. Almost a third of the facilities in Malawi have an excellent retention level. However, high early LTF found in many other facilities is of concern and underlines the pressing need to understand the barriers to retention in care for women who start ART under Option B+. Our findings support the need for innovative service delivery models like community or family-based approaches and interventions to promote retention such as motivating text messages [32], systematic defaulter tracing or improving confidentiality in PMTCT. LTF was particularly high in facilities with a high patient volume and in patients who start ART during pregnancy on the day of HIV diagnosis - when policy makers plan improvements to Option B+, they should therefore pay particular attention to these patients.

\section{Acknowledgements}

We thank all patients, doctors and study nurses in the participating facilities, and Lynne Mofenson, MD, from the National Institute of Child Health and Human Development for helpful comments. We thank Kali Tal, $\mathrm{PhD}$ from the Institute of Social and Preventive Medicine, University of Bern, for editing the manuscript.

L.T., A.H. and O.K. had full access to all the data in the study. A.H. and L.T. take responsibility for the integrity of the data and the accuracy of the data analysis. O.K. had the final responsibility in deciding to submit for publication. 
Author contributions: L.T., A.H., G.W. and O.K. designed and coordinated the analyses. A.H., O.K., L.T. did statistical analyses. M.Z. advised on statistical analyses. A.H., L.T., G.W., O.K. wrote the first draft of the paper. A.J., F.C., Z.C., J.Jv.O., H.T. and S.P. assisted in implementation, fieldwork and data collection at study facilities and contributed to the writing of the article. L.M. contributed to the writing of the article. A.H., H.T., LT extracted and managed the data. A.B., F.V. and W.N. helped with data extraction and management. All authors contributed to the final text.

This work was supported by the National Institute of Allergy and Infectious Diseases (NIAID) and the Eunice Kennedy Shriver National Institute for Child Health and Human Development (NICHD) [IeDEA Southern Africa, grant number U01 AI069924], the Bill and Melinda Gates Foundation and the USAID-NIH initiative http://sites.nationalacademies.org/PGA/dsc/ peerhealth/index.htm Partnerships for Enhanced Engagement in Research (PEER) Health (NIH/PEER) [grant number AID-OAA-A-11-00012]. The content is solely the responsibility of the authors and does not necessarily represent the official views of the sponsors. O.K. was supported by a PROSPER fellowship grant from the Swiss National Science Foundation. L.M. is supported by the Elizabeth Glaser Pediatric AIDS Foundation. The Malawi MOH HIV/ AIDS Programme is funded by The Global Fund and President's Emergency Plan for AIDS Relief (PEPFAR).

The funders did not influence the study design, data collection, data analysis, data interpretation or writing of the report.

\section{Conflicts of interest}

The authors declare that they have no conflicts of interest.

\section{References}

1. World Health Organisation, PMTCT strategic vision 20102015. Preventing mother-to-child transmission of HIV to reach the UNGASS and Millennium Development Goals 2010. http:// www.who.int/hiv/pub/mtct/strategic_vision/en/. [Accessed 1 May 2013]

2. World Health Organisation. Antiretroviral drugs for treating pregnant women and preventing HIV infection in infants. Recommendations for a public health approach 2010 revision. http://whqlibdoc.who.int/publications/2010/ 9789241599818 eng.pdf. [Accessed 1 May 2013]

3. Mofenson L. Prevention in neglected subpopulations: prevention of mother-to-child transmission of HIV infection. Clin Infect Dis 2010; 50 (Suppl 3):130-148.

4. Schouten EJ, Jahn A, Chimbwandira F, Harries AD, Van Damme W. Is Option B+ the best choice? Lancet 2013; 381:12721273.

5. Wettstein C, Mugglin C, Egger M, Blaser N, Vizcaya LS, Estill J, et al. Missed opportunities to prevent mother-to-childtransmission: systematic review and meta-analysis. AIDS 2012; 26:2361-2373.
6. Government of Malawi Ministry of Health 2012. Integrated HIV Program Report July - September. http://www. hivunitmohmw. org/uploads/Main/Quarterly_HIV_Programme_Report_2012_ Q3.pdf. [Accessed 1 May 2013]

7. Schouten EJ, Jahn A, Midiani D, Makombe SD, Mnthambala A, Chirwa Z, et al. Prevention of mother-to-child transmission of HIV and the health-related Millennium Development Goals: time for a public health approach. Lancet 2011; 378:282-284.

8. Coutsoudis A, Goga A, Desmond C, Barron P, Black V, Coovadia $\mathrm{H}$. Is Option B + the best choice? Lancet 2013; 381:1273.

9. Kellerman S, Jay JS, Quick JD. Is Option B+ the best choice? Lancet 2013; 381:1272.

10. Goosby EP. Is Option B+ the best choice? Lancet 2013; 381:1272.

11. Hirnschall G, Doherty $M$, Shaffer N. Is Option B+ the best choice? Lancet 2013; 381:1271-1272.

12. World Health Organisation. Consolidated guidelines on the use of antiretroviral drugs for treating and preventing HIV infection: recommendations for a public health approach. June 2013. http://apps.who.int/iris/bitstream/10665/85321/1/ 9789241505727_eng.pdf. [Accessed 13 August 2013]

13. Hargrove JW, Humphrey JH, ZVITAMBO Study Group. Mortality among HIV-positive postpartum women with high CD4 cell counts in Zimbabwe. AIDS 2010; 24:F11-14.

14. Cohen MS, Chen YQ, McCauley M, Gamble T, Hosseinipour MC, Kumarasamy N, et al. Prevention of HIV-1 infection with early antiretroviral therapy. N Engl / Med 2011; 365:493-505.

15. WHO, UNICEF, UNAIDS. Global update on HIV treatment 2013: Results, impact and opportunities. June 2013. http:// apps.who.int/iris/bitstream/10665/85326/1/ 9789241505734_eng.pdf. [Accessed 13 August 2013]

16. Centers for Disease Control and Prevention, (CDC). Impact of an innovative approach to prevent mother-to-child transmission of HIV 2013: Malawi, July 2011-September 2012. Morb Mortal Wkly Rep 2013; 62:148-151.

17. World Health Organisation. Antiretroviral therapy for HIV infection in adults and adolescents. Recommendations for a public health approach. 2006 revision. http://www.who.int/hiv/ pub/arv/adult/en/. [Accessed 1 May 2013]

18. Government of Malawi Ministry of Health (2012) Integrated HIV Program Report April-June 2012. http://www.hivunitmohmw. org/uploads/Main/Quarterly_HIV_Programme_Report_2012_ Q2.pdf. [Accessed 1 May 2013]

19. Douglas GP, Gadabu OJ, Joukes S, Mumba S, McKay MV, Ben-Smith $A$, et al. Using touchscreen electronic medical record systems to support and monitor national scale-up of antiretroviral therapy in Malawi. PLoS Med 2010; 7: pii: e1000319. doi: 10.1371/journal.pmed.1000319.

20. Lau B, Cole SR, Gange SJ. Competing risk regression models for epidemiologic data. Am / Epidemiol 2009; 170:244-256.

21. Fine JP, Gray RJ. A proportional hazards model for the subdistribution of a competing risk. I Am Stat Assoc 1999; 94:496509.

22. Cornell M, Grimsrud A, Fairall L, Fox MP, van Cutsem G, Giddy $J$, et al. Temporal changes in programme outcomes among adult patients initiating antiretroviral therapy across South Africa, 2002-2007. AIDS 2010; 24:2263-2270.

23. Speight $C$, Phiri S, Hosseinipour $M$, Tweya H, Chimbwandira F, Chikonda J, et al. Implementing Option B+ for prevention of mother to child transmission at Bwaila Maternity Unit, Lilongwe: the first 18 months. Presented at the 7th IAS Conference on HIV Pathogenesis, Treatment and Prevention. Kuala Lumpur, Malaysia.

24. Hardon A, Vernooij E, Bongololo-Mbera G, Cherutich P, Desclaux A, Kyaddondo D, et al. Women's views on consent, counseling and confidentiality in PMTCT: a mixed-methods study in four African countries. BMC Public Health 2012; 12:26 doi: $10.1186 / 1471-2458-12-26$.

25. Gebrekristos HT, Mlisana KP, Karim Q. Patients' readiness to start highly active antiretroviral treatment for HIV. BMJ 2005; 331:772-775.

26. Orrell C. Antiretroviral adherence in a resource-poor setting. Curr HIVIAIDS Rep 2005; 2:171-176.

27. Medley A, Garcia-Moreno C, McGill S, Maman S. Rates, barriers and outcomes of HIV serostatus disclosure among women in developing countries: implications for prevention of mother-to-child transmission programmes. Bull World Health Org 2004; 82:299-307. 
28. Anglewicz $\mathrm{P}$, Chintsanya J. Disclosure of HIV status between spouses in rural Malawi. AIDS Care 2011; 23:998-1005.

29. Myer L, Zulliger R, Bekker LG, Abrams E. Systemic delays in the initiation of antiretroviral therapy during pregnancy do not improve outcomes of HIV-positive mothers: a cohort study. BMC Pregnancy Childbirth 2012; 12:94. doi: 10.1186/14712393-12-94.

30. Brinkhof MW, Spycher BD, Yiannoutsos C, Weigel R, Wood R Messou $E$, et al. Adjusting mortality for loss to follow-up: analysis of five ART programmes in sub-Saharan Africa. PloS One 2010; 5:e14149.
31. Tweya H, Gareta D, Chagwera F, Ben-Smith A, Mwenyemasi J, Chiputula $F$, et al. Early active follow-up of patients on antiretroviral therapy (ART) who are lost to follow-up: the 'Backto-Care' project in Lilongwe, Malawi. Trop Med Int Health 2010; 15 (Suppl 1):82-89.

32. Joseph-Davey D, Ponce W, Augusto O, Traca D, Jetha E, de Palha de Sousa C. Improved retention in HIV care following SMS reminders in Mozambique: a randomized controlled trial. Presented at the 7th IAS Conference on HIV Pathogenesis, Treatment and Prevention. Kuala Lumpur, Malaysia. 